\title{
Bilateral radioiodine uptake by the non-lactating breast of a single nulliparous woman: a case report and literature review
}

Masoud Moslehi' ${ }^{1}$ Majid Assadi² 1Department of Nuclear Medicine, Seyedoshohada Hospital, Isfahan University of Medical
Sciences, Iran
2The Persian Gulf Nuclear Medicine Research Center, Bushehr University of Medical
Sciences, Iran

Submitted: 15 December 2010

Accepted: 28 April 2011

Arch Med Sci 2012; 8, 3: 575-577

DOI: 10.5114 /aoms.2012.28592

Copyright (c) 2012 Termedia \& Banach

\section{Corresponding author:} Majid Assadi MD

The Persian Gulf Nuclear Medicine Research Center Bushehr University of Medical Sciences Bushehr 3631, Iran Phone: +98-771-2580169 Fax: +98-771-2541828 E-mail: assadipoya@yahoo.com, asadi@bpums.ac.ir
Whole-body I-131 scintigraphy (I-131 WBS) has been a helpful diagnostic procedure for evaluating the existence of residual thyroid bed remnants as well as possible metastasis in patients with differentiated thyroid cancer after surgery $[1,2]$. In the interpretation of I-131 WBS, breast uptake may be misread as thyroid cancer lung metastasis if it occurs with an atypical pattern or is clinically unpredictable [3]. Radioiodine uptake by women who are lactating [4], have a history of previous lactation [5], hyperprolactinaemia [3], or a pathologic cause [1, 6-8] is well documented; however, bilateral radioiodine uptake by the non-lactating breast of single women with negative examination for galactorrhoea has rarely been reported [5].

We report a case of bilateral breast uptake on low-dose 131-I wholebody scintigraphy in a thyroid cancer patient with a negative history of breastfeeding and also negative galactorrhoea upon examination.

An 18-year-old Asian woman was referred to our centre for follow-up after a near-total thyroidectomy for histologically confirmed papillary thyroid cancer with no signs of extra-thyroidal extension (stage T2NOMO). The patient had received $3700 \mathrm{MBq}^{131}$ I one year earlier.

At this time, following withdrawal from levothyroxine 4 weeks earlier (TSH > $50 \mathrm{mU} / \mathrm{l}$; normal values: $0.2-5 \mathrm{mU} / \mathrm{l}$ ) and administration of $185 \mathrm{MBq}$ ${ }^{131}$, whole-body scintigraphy (WBS) was performed. The WBS scan was performed on a rotating digital gamma camera equipped with a highenergy all-purpose parallel-hole collimator. The WBS showed increased activity in the anterior aspect of the chest, with additional lateral projections suggesting uptake by both breasts (Figure 1). Breast uptake was mild (Figure $1 \mathrm{~A}$ ) compared with mediastinal uptake. Serum thyroglobulin (Tg) level was below $1 \mathrm{ng} / \mathrm{ml}$. Prolactin level was within normal limits and she had no clinical history of either galactorrhoea or mastitis and was not clinically suspected of having a pituitary or suprasellar tumour. During 1-year follow-up, the general condition of the patient was good and also the follow-up breast ultrasonography was unremarkable.

Whole-body ${ }^{131}$ scintigraphy has been a helpful diagnostic procedure for evaluating the existence of residual thyroid bed remnants as well as possible metastases in patients with differentiated thyroid cancer after surgery [1]. Breast uptake may be misread as metastases of thyroid cancer in the lungs [3]. Radioiodine uptake by women who are lactating [4], or 


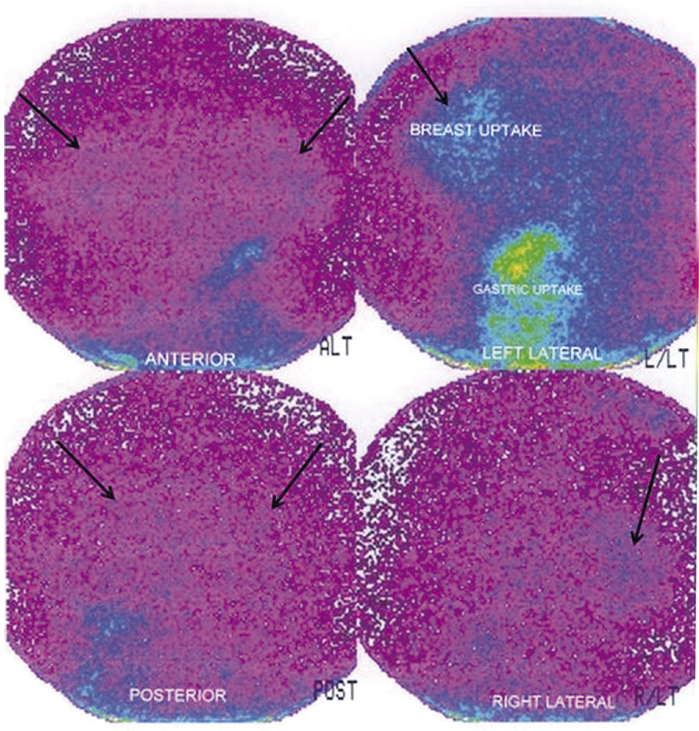

Figure 1. (Left upper) Anterior and (left lower) posterior projections of the ${ }^{131}$ whole body scan show mild bilateral 1311 uptake in the lower chest region compared with the mediastinum. (Right lower) Right and (right upper) left lateral projections of the chest confirm that the ${ }^{131}$ activity is in the bilateral breast regions

have a history of previous lactation [5], or hyperprolactinaemia [3], or other pathological causes such as benign breast diseases $[1,6,7]$, is well documented; however, bilateral ${ }^{131}$ I uptake by the nonlactating breasts of single women with negative examination for galactorrhoea has rarely been reported [5].

Radioiodine uptake within the mammary gland has been reported in both lactating and non-lactating women $[4,5]$. There are four different patterns of breast uptake, full, focal, crescentic, and irregular, and uptake may be unilateral or bilateral [9]. Additional lateral imaging with the breast suspended was helpful for diagnosis. In addition, a discrepancy between ${ }^{131}$ I and ${ }^{201} \mathrm{Tl}$ uptake in the chest region may help to diagnose non-malignant breast uptake of ${ }^{131}$ [ [3].

The mechanism of breast ${ }^{131} \mid$ accumulation remains uncertain. In most studies, the prolactin level is considered a major factor which may be induced by various drugs [10] or by prolactinoma [3]. In addition, hypothyroidism, developed in preparation for ${ }^{131}$ I scanning and treatment, can induce mild hyperprolactinaemia, with or without galactorrhoea [5].

Others have evaluated 23 non-pregnant women with breast uptake of ${ }^{131} \mathrm{l}$, including 4 single nulliparous patients, 3 postmenopausal patients, and 16 other cases [5]. The mean time from discontinuation of breastfeeding was 11.4 months. They found no remarkable differences in pattern of breast uptake in patients with normal and elevated prolactin levels, suggesting that prolactin may not play a principal role in breast ${ }^{131}$ I uptake [5]. In addition, they showed that sensitization of breast tissue to prolactin due to previous prolonged periods of lactation was an unlikely explanation, because there were no consistent changes in the pattern or the relative intensity of breast uptake after a mean follow-up period of 11.4 months [5]. Furthermore, breast uptake was seen in four single nulliparous women who had never breastfed and one of these had a normal prolactin level and no galactorrhoea upon clinical examination, as in our case. In addition, neither the dose or kind of radioiodine isotope nor the scanning time appeared to be an important factor for radioiodine breast uptake [5]. Therefore, the breast activity in our case might be idiopathic in origin.

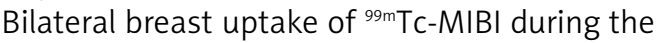
menstrual cycle and in the postmenopausal course has been reported, with $58 \%$ and $72 \%$ of the cases in the first and fourth weeks of the menstrual cycle, respectively, and with $5 \%$ of postmenopausal women studied [11]. Our patient was in the fourth week of her menstrual cycle. Nevertheless, whether changes in hormonal levels during the menstrual cycle have any effect on ${ }^{131}$ l breast uptake requires further studies.

In conclusion, this case showed bilateral uptake of ${ }^{131} \mathrm{I}$ in a single nulliparous woman who had never breastfed; that could be misinterpreted as metastases. So, physicians should be aware of this possible source of confusion even in this subgroup. A high degree of suspicion, accompanied by careful attention, is needed for proper interpretation of positive radioiodine scans.

\section{References}

1. Allen T, Wiest P, Vela S, Hartshorne M, Crooks LA. I-131 uptake in the breast for thyroid cancer surveillance with biopsyproven benign tissue. Clin Nucl Med 1998; 23: 585-7.

2. Oszukowska L, Knapska-Kucharska M, Lewiński A. Effects of drugs on the efficacy of radioiodine (I) therapy in hyperthyroid patients. Arch Med Sci 2010; 6: 4-10.

3. Kao PF, Chang HY, Tsai MF, Lin KJ, Tzen KY, Chang CN. Breast uptake of iodine-131 mimicking lung metastases in a thyroid cancer patient with a pituitary tumour. $\mathrm{Br}$ J Radiol 2001; 74: 378-81.

4. Brzozowska M, Roach PJ. Timing and potential role of diagnostic I-123 scintigraphy in assessing radioiodine breast uptake before ablation in postpartum women with thyroid cancer: a case series. Clin Nucl Med 2006; 31: 683-7.

5. Hammami MM, Bakheet S. Radioiodine breast uptake in nonbreastfeeding women: clinical and scintigraphic characteristics. J Nucl Med 1996; 37: 26-31.

6. Serafini A, Sfakianakis G, Georgiou M, Morris J. Breast cyst simulating metastases on iodine-131 imaging in thyroid carcinoma. J Nucl Med 1998; 39: 1910-2.

7. Bakheet SM, Powe J, Hammami MM. Radioiodine uptake in the chest. J Nucl Med 1997; 38: 984-6.

8. Hosseini M, Houshmand M, Ebrahimi A. Breast cancer risk not only was not associated with CYP17/A2 allele but also was related to A1 allele. Arch Med Sci 2009; 5: 103-6. 
9. Bakheet SM, Hammami MM. Patterns of radioiodine uptake by the lactating breast. Eur J Nucl Med 1994; 21: 604-8.

10. Ronga G, Bruno R, Puxeddu E, et al. Radioiodine uptake in non-lactating mammary glands: evidence for a causative role of hyperprolactinemia. Thyroid 2007; 17: 363-6.

11. Thet-Thet-Lwin, Takeda T, Wu J, et al. Diffuse and marked breast uptake of both 123I-BMIPP and 99mTc-TF by myocardial scintigraphy. Ann Nucl Med 2000; 14: 315-8. 